\title{
Measurement of the top-antitop mass difference (CMS)
}

\author{
Gerrit Van Onsem* \\ On behalf of the CMS Collaboration \\ Vrije Universiteit Brussel \\ E-mail: Gerrit.Patrick.Van. Onsemecern.ch
}

\begin{abstract}
The standard model of particle physics is a local gauge-invariant quantum field theory in which the CPT symmetry plays a fundamental role. One of the consequences of CPT invariance is an equal mass for a particle and its antiparticle. In order to test the CPT invariance, we performed a measurement of the mass difference between the top and the antitop quark $\left(\Delta m_{\mathrm{t}}=m_{\mathrm{t}}-m_{\overline{\mathrm{t}}}\right)$ using events with a muon or an electron and at least four jets in the final state. The analysis is based on data collected by the CMS experiment at the LHC, corresponding to an integrated luminosity of $4.96 \pm 0.11 \mathrm{fb}^{-1}$, and yields the value of $\Delta m_{\mathrm{t}}=-0.44 \pm 0.46$ (stat.) \pm 0.27 (syst.) GeV. This result is consistent with equality of particle and antiparticle masses required by CPT invariance, and provides a significantly improved precision relative to existing measurements.
\end{abstract}

36th International Conference on High Energy Physics,

July 4-11, 2012

Melbourne, Australia

${ }^{*}$ Speaker. 


\section{Introduction and overview}

Many predictions of the standard model of particle physics have been confirmed by previous experiments, consolidating its theoretical foundation as a local gauge-invariant quantum field theory. Symmetries such as charge conjugation $(\mathrm{C})$, parity or space reflection $(\mathrm{P})$ and time reversal (T) play a key role in the standard model, and the combined CPT symmetry appears to be conserved in nature. A major consequence of CPT conservation is an equal mass for a particle and its antiparticle. Since top quarks have a short lifetime and decay before hadronization, (anti)top quark events provide an excellent opportunity to test the invariance of the CPT symmetry. The mass difference between the top quark and the antitop quark was measured previously by the D0 and CDF experiments, and showed no significant deviation from zero [1, 2, 3].

We performed a measurement of the top-antitop mass difference using top quark pair events produced in proton-proton collisions at $\sqrt{s}=7 \mathrm{TeV}$, recorded with the Compact Muon Solenoid (CMS) detector at the Large Hadron Collider (LHC). We select top quark pair events with one hadronically decaying $\mathrm{W}$ boson ( $\mathrm{t} \rightarrow \mathrm{bW}^{+} \rightarrow \mathrm{bq}^{\prime}$, or its charge conjugate) and one leptonically decaying $\mathrm{W}$ boson ( $\mathrm{t} \rightarrow \mathrm{bW}^{+} \rightarrow \mathrm{b}^{+} v_{\ell}$, or its charge conjugate), where the lepton $\ell$ is a muon or an electron. Next, the data is split in negatively charged and positively charged lepton samples that contain three-jet decays of the associated top or antitop quarks, respectively. We apply the Ideogram likelihood method [4] to measure the mass of the top quark $m_{\mathrm{t}}$ and the antitop quark $m_{\bar{t}}$, and the difference between the masses in the two distinct samples of lepton charge is taken as the mass difference $\Delta m_{\mathrm{t}}=m_{\mathrm{t}}-m_{\overline{\mathrm{t}}}$. The procedure includes a kinematic fit of the events to a $\overline{\mathrm{t}}$ hypothesis where we only consider the top or antitop quark that decays to three jets.

\section{Event reconstruction and selection}

A detailed description of the CMS detector can be found in Ref. [5]. Events are reconstructed using the particle-flow (PF) algorithm [6], which combines the information of all CMS sub-detectors used to identify individual muons, electrons, photons, charged and neutral hadrons. The list of charged and neutral PF particles originating from the primary collision vertex is used as input for an anti- $k_{\mathrm{T}}$ jet-clustering algorithm with a distance parameter of 0.5. Isolated muons and electrons are excluded from the jet clustering. Jet-energy-scale corrections are applied to all the jets in data and simulation.

We select events with at least four jets with transverse momentum $\left(p_{\mathrm{T}}\right)$ larger than $30 \mathrm{GeV}$ and pseudorapidity $(|\eta|)$ smaller than 2.4. In the $\mu+$ jets (e+jets) channel we require one isolated muon (electron) with $p_{\mathrm{T}}>20 \mathrm{GeV}$ and $|\eta|<2.1\left(p_{\mathrm{T}}>30 \mathrm{GeV}\right.$ and $\left.|\eta|<2.5\right)$. This selection is optmized to select semi-leptonic $t \bar{t}$ events, and the main background events are coming from $\mathrm{W}+\mathrm{jets}, \mathrm{Z} / \gamma^{*}+\mathrm{jets}$, single-top and multijet processes. The kinematic properties of the multijet background are estimated from a data sample of events that pass all selections, but with an inverted lepton-isolation criterion. The $t \bar{t}$ signal is modeled using the MadGraph generator [7] interfaced to PYTHIA [8], and the background process events are generated with MadGraph or POWHEG [9].

\section{Kinematic fit and Ideogram method}

A kinematic fit of $\ell+\mathrm{jets}$ final states to a $\mathrm{t} \overline{\mathrm{t}}$ hypothesis is used to reconstruct the mass of the 

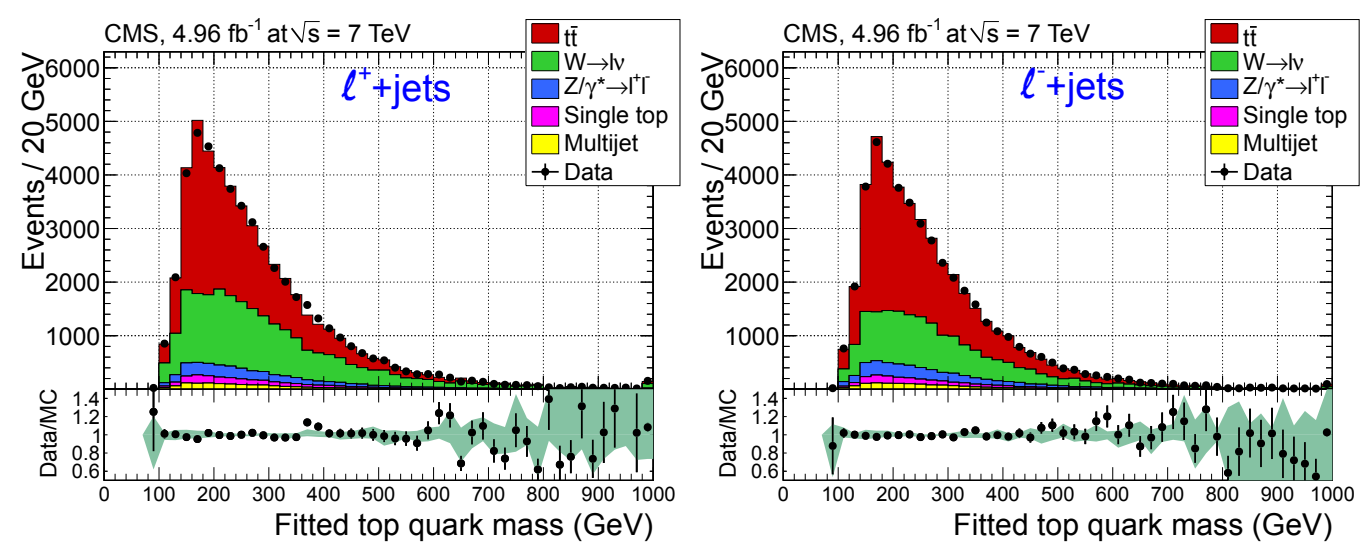

Figure 1: Data and simulation for the fitted top-quark mass distributions agree very well, both in the $\ell^{+}+$jets (left) and $\ell^{-}+$jets (right) samples.

hadronically decaying top quark. For each event, we consider the four highest- $p_{\mathrm{T}}$ jets, hence there are 12 possible jet-quark assignments. For each jet combination, the jet energies first get corrected to the parton level. These jet energy corrections are derived from simulation, for light-quark and b-quark jets seperately, and in bins of $p_{\mathrm{T}}$ and $|\eta|$. Next, the momenta of the two jets assigned to the quarks coming from the W-boson decay are varied within their resolutions, using the mass of the $\mathrm{W}$ boson as a constraint. In this way the reconstruction of the mass of the three-jet decay of the top quark $\left(\mathrm{t} \rightarrow \mathrm{bW}^{+} \rightarrow \mathrm{bq} \overline{\mathrm{q}}^{\prime}\right)$ is modified. The jet combinations $i$ with $\chi_{i}^{2}<10$ correspond to the fitted top-quark mass $m_{i}$. The most important gain from the kinematic fit is that it improves the resolution on the top-quark mass. The fitted top-quark mass distributions in the $\ell^{+}+$jets and $\ell^{-}+$jets samples are shown in Figure 1.

The fitted values of the top-quark mass $m_{i}$, the uncertainty on the mass $\sigma_{i}$ and the $\chi_{i}^{2}$, obtained for each combination of jets $i$, are used as input to the Ideogram method. For every event a likelihood is calculated:

$$
\mathscr{L}_{\text {event }}\left(x ; y \mid m_{\mathrm{t}}\right)=f_{\overline{\mathrm{t}} \mathrm{t}} P_{\mathrm{t} \overline{\mathrm{t}}}\left(x ; y \mid m_{\mathrm{t}}\right)+\left(1-f_{\mathrm{tt}}\right) P_{\mathrm{bkg}}(x),
$$

where $P_{\mathrm{t}}\left(x ; y \mid m_{\mathrm{t}}\right)$ and $P_{\mathrm{bkg}}(x)$ are the probability densities for $\mathrm{t} \overline{\mathrm{t}}$ and background events, respectively. The set of observables $x$ represents the number of b-tagged jets $n_{\mathrm{b}}$, the lepton charge $q^{\ell}$ and the fitted top quark masses $m_{i}$, while the quantity $y$ represents $\sigma_{i}$ and $\chi_{i}^{2}$. The number of b-tagged jets and the lepton charge are assumed to be uncorrelated with the mass information in a given event. In this way the signal and background probability densities can be treated as the product of the probability to observe $n_{\mathrm{b}} \mathrm{b}$ jets, the probability to observe a certain lepton charge $q^{\ell}$, and the probability to observe a set of masses $m_{i}$. The measured top-quark mass and its statistical uncertainty are extracted from the combined likelihood of the full sample, which is the product of the individual likelihoods of all the events.

\section{Calibration of individual mass measurements}

Since the likelihood defined in the Ideogram method is a simplified model, we have to correct for a possible bias of the estimated mass and the estimated statistical uncertainty. Therefore a cal- 


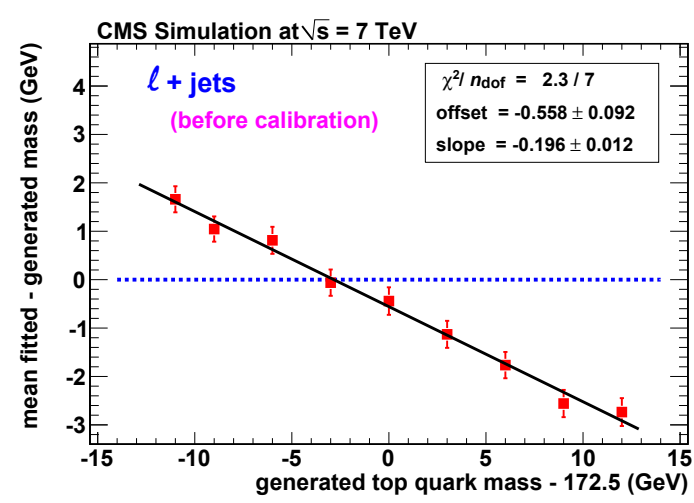

Figure 2: The bias on the estimated top quark mass depends linearly on the generated top quark mass for $\ell+$ jets events.

ibration of the procedure is performed using pseudo-experiments. For this purpose, we simulated nine samples of $t \bar{t}$ events, for top-quark masses between $161.5 \mathrm{GeV}$ and $184.5 \mathrm{GeV}$. Figure 2 shows the bias on the estimated top-quark mass as a function of generated mass for the combined $\ell^{+}$and $\ell^{-}$events. We can correct for this bias using the fitted linear calibration. The width of the pull distribution is also found to be slightly larger than one, therefore the statistical uncertainties on the final mass measurement are scaled up by about $16 \%$.

\section{Measurement of the top-antitop mass difference}

We apply the analysis seperately to $\ell^{-}+$jets events and $\ell^{+}+$jets events, and take the difference of the two extracted top quark mass values: $\Delta m_{\mathrm{t}}=m_{\mathrm{t}}^{\text {Ideogram }}-m_{\overline{\mathrm{t}}}^{\text {Ideogram }}$. In the $\mu+$ jets channel, we obtain a mass difference $\Delta m_{\mathrm{t}}=0.13 \pm 0.61$ (stat.) $\mathrm{GeV}$, while in the e+jets channel, we obtain $\Delta m_{\mathrm{t}}=-1.28 \pm 0.70$ (stat.) GeV. Combining the $\mu+$ jets and e+jets samples, we measure

$$
\Delta m_{\mathrm{t}}=-0.44 \pm 0.46 \text { (stat.) } \mathrm{GeV} \text {. }
$$

This result is compatible with the expectation from the hypothesis of CPT symmetry, even ignoring systematic uncertainties. The average fitted top-quark mass of $m_{\mathrm{t}}=173.36 \pm 0.23$ (stat.) GeV is also in agreement with other measurements $[10,11,12,13]$.

\section{Systematic uncertainties}

Many systematic uncertainties relevant for the absolute measurement of $m_{\mathrm{t}}$ are reduced in the context of our measurement, as these systematic effects would alter the measured properties of top and antitop quarks in a similar and correlated way. Several sources of systematic uncertainties are considered in the analysis, and their effect is summarized in Table 1. The jet energy scale contributes to the uncertainty since top and anti-top quarks at the LHC are produced with slightly different rapidity distributions, so an $\eta$-dependence for the jet response can lead to a residual effect on the measurement of $\Delta m_{\mathrm{t}}$. The asymmetry of the background composition can cause an additional residual effect, due to the $\eta$-dependence of the jet energy resolution uncertainties. The relative jet 
energy scale for $\mathrm{b}$ and $\overline{\mathrm{b}}$ jets is also expected to contribute as a source of systematic uncertainty, since the simulation describes differences in the fragmentation of $\mathrm{b}$ and $\bar{b}$ jets. The $t \bar{t}$ signal fraction $f_{\mathrm{t}}$, the different background composition for $\mathrm{W}+\mathrm{jets}$ events in the $\ell^{+}$and $\ell^{-}$samples, and the relative background compositions all have an effect in the top-quark mass estimation in the Ideogram method, and have associated uncertainties. The uncertainty in the pileup modeling and pileup reweighting of simulated events used in the analysis is evaluated as well, and no significant effects of the number of pileup events on the measurement is observed. The b-tagging efficiency can affect the impact of background processes on the mass estimation, and a possible difference in mass bias between $\ell^{+}+$jets and $\ell^{-}+$jets would affect the method calibration itself. Finally, the parton distribution functions are included in the estimation of the total systematic uncertainty because they determine for instance the difference in production of $\mathrm{W}^{+}$and $\mathrm{W}^{-}$events.

Table 1: Overview of the systematic uncertainties on $\Delta m_{\mathrm{t}}$. The total is obtained by adding in quadrature the contributions from all sources, by choosing for each the larger of the estimated shift or its statistical uncertainty, as indicated in bold.

\begin{tabular}{lc}
\hline \hline Source & Estimated effect $(\mathrm{GeV})$ \\
\hline Jet energy scale & $0.04 \pm \mathbf{0 . 0 8}$ \\
Jet energy resolution & $0.04 \pm \mathbf{0 . 0 6}$ \\
b vs. $\bar{b}$ jet response & $\mathbf{0 . 1 0} \pm 0.10$ \\
Signal fraction & $\mathbf{0 . 0 2} \pm 0.01$ \\
Difference in $\mathrm{W}^{+} / \mathrm{W}^{-}$production & $\mathbf{0 . 0 1 4} \pm 0.002$ \\
Background composition & $\mathbf{0 . 0 9} \pm 0.07$ \\
Pileup & $\mathbf{0 . 1 0} \pm 0.05$ \\
b-tagging efficiency & $\mathbf{0 . 0 3} \pm 0.02$ \\
b vs. $\bar{b}$ tagging efficiency & $\mathbf{0 . 0 8} \pm 0.03$ \\
Method calibration & $0.11 \pm \mathbf{0 . 1 4}$ \\
Parton distribution functions & $\mathbf{0 . 0 8 8}$ \\
\hline Total & 0.27 \\
\hline \hline
\end{tabular}

\section{Summary}

We measured the mass difference between the top quark and the antitop quark with the Ideogram method, using the lepton+jets top quark pair event sample collected by the CMS experiment, with an integrated luminosity of $4.96 \pm 0.11 \mathrm{fb}^{-1}$. This yields the most precise measurement to date of this quantity:

$$
\Delta m_{\mathrm{t}}=-0.44 \pm 0.46 \text { (stat.) } \pm 0.27 \text { (syst.) GeV. }
$$

The measured value is in agreement with the consequence of CPT invariance, which requires no mass difference. More details can be found in [14]. 


\section{References}

[1] D0 Collaboration, "Direct measurement of the mass difference between top and antitop quarks", Phys. Rev. Lett. 103 (2009) 132001.

[2] CDF Collaboration, "Measurement of the mass difference between $t$ and $\bar{t}$ quarks", Phys. Rev. Lett. 106 (2011) 152001.

[3] D0 Collaboration, "Direct measurement of the mass difference between top and antitop quarks", Phys. Rev. D. 84 (2011) 052005.

[4] DELPHI Collaboration, "Measurement of the Mass and Width of the $\mathrm{W}$ boson in $e^{+} e^{-}$Collisions at $\sqrt{s}=161$ - 209 GeV”, Eur. Phys. J. C 55 (2008) 1-38.

[5] CMS Collaboration, “The CMS experiment at the CERN LHC”, JINST 03 (2008) S08004.

[6] CMS Collaboration, "Commissioning of the Particle-Flow Reconstruction in Minimum-Bias and Jet Events from pp Collisions at 7 TeV”, CMS-PAS-PFT-10-002 (2010), http://cdsweb.cern.ch/record/1279341.

[7] J. Alwall, M. Herquet, F. Maltoni et al., "MadGraph 5 : Going Beyond”, JHEP 06 (2011) 128.

[8] T. Sjöstrand, S. Mrenna, and P. Skands, "PYTHIA 6.4 Physics and Manual”, JHEP 05 (2006) 026.

[9] S. Frixione, P. Nason and C. Oleari, "Matching NLO QCD computations with parton shower simulations: the POWHEG method", JHEP 11 (2007) 070.

[10] CMS Collaboration, "Measurement of the top-quark pair-production cross section and the top-quark mass in the dilepton channel at $\sqrt{s}=7$ TeV”, JHEP 07 (2011) 049.

[11] ATLAS Collaboration, "Measurement of the top quark mass with the template method in the top antitop $\rightarrow$ lepton + jets channel using ATLAS data", Eur. Phys. J. C 72 (2012) 2046.

[12] CDF Collaboration, “Top Quark Mass Measurement in the Lepton + Jets Channel Using a Matrix Element Method and in situ Jet Energy Calibration”, Phys. Rev. Lett. 105 (2010) 252001.

[13] D0 Collaboration, "Precise measurement of the top-quark mass from lepton+jets events at D0", Phys. Rev. D 84 (2011) 032004.

[14] CMS Collaboration, "Measurement of the mass difference between top and antitop quarks", accepted by JHEP (2012), http://arxiv.org/abs/1204.2807 